\title{
Pseudoaneurisma de arteria temporal superficial traumática
}

\author{
Traumatic superficial temporal artery pseudoaneurysm
}

\author{
Brian Domínguez González, ${ }^{*}$ Xavier Arroyo Sámano, ${ }^{*}$ Carolina Aragón Prieto, ${ }^{*}$ \\ Óscar Vera Díaz, ${ }^{\ddagger}$ Carlos Flores Ramírez, ${ }^{*}$ Carlos Eduardo Diéguez Campa ${ }^{\S}$
}

Citar como: Domínguez GB, Arroyo SX, Aragón PC, Vera DÓ, Flores RC, Diéguez CCE . Pseudoaneurisma de arteria temporal superficial traumática. Acta Med Grupo Angeles. 2021; 19 (2): 267-271. https://dx.doi.org/10.35366/100453

\section{Resumen}

El pseudoaneurisma de la arteria temporal superficial fue descrito en 1740 por Thomas Bartholin. Se han documentado cerca de 400 casos en la literatura. Los pseudoaneurismas son dados por la disrupción de la continuidad de la pared arterial, y el trauma es su primer factor causal. La mayoría de los pacientes presentan el pseudoaneurisma dentro de dos a seis semanas posteriores al traumatismo; la fístula arteriovenosa es la complicación más frecuente. Se trata de paciente masculino de 37 años, sin antecedentes de importancia, inicia su padecimiento tres meses antes de su interrogatorio luego de sufrir agresión por terceras personas en región frontotemporal derecha, con presencia posterior de masa pulsátil indolora en trayecto de arteria temporal superficial, la cual, según refirió, fue aumentando de tamaño de manera progresiva. EI diagnóstico clínico de pseudoaneurisma de la arteria temporal superficial se sospechó y se confirmó con angiotomografía. El pseudoaneurisma es una entidad infrecuente, pocas veces reportada en la literatura, por lo tanto subdiagnosticada, debe sospecharse siempre ante una masa pulsátil con antecedente de un evento traumático.

Palabras clave: Pseudoaneurisma, arteria temporal, traumatismo.

\section{Abstract}

The superficial temporal artery pseudoaneurysm was described in 1740 by Thomas Bartholin; about 400 cases have been documented in the literature. Pseudoaneurysms are caused by disruption of the continuity of the arterial wall, trauma is its first causal factor. Most patients present with a pseudoaneurysm within two to six weeks after the trauma, with the arteriovenous fistula being the most frequent complication. A 37-year-old male patient, without a significant history, began his condition three months before his interrogation, after suffering aggression by third parties in the right frontotemporal region, with the subsequent presence of a painless pulsatile mass in the superficial temporal artery path, which he reported it was gradually increasing in size. The clinical diagnosis of pseudoaneurysm of the superficial temporal artery was suspected and confirmed with angio-tomography. Pseudoaneurysm is an infrequent entity, rarely reported in the literature, therefore underdiagnosed, it should always be suspected in a pulsatile mass with a history of traumatic event.

Keywords: Pseudoaneurysm, temporary artery, trauma.

* Servicio de Angiología y Cirugía Vascular, Hospital Central del Estado de Chihuahua.

₹ Servicio de Angiología y Cirugía Vascular, Hospital General de Ciudad Juárez.

$\S$ Instituto de Ciencias Biomédicas. Universidad Autónoma de Ciudad Juárez. Departamento de Neuroquímica. Instituto Nacional de Neurología y Neurocirugía.

Aceptado: 18-05-2020.

Correspondencia: Brian Domínguez González Correo electrónico: bbdg114@hotmail.com 


\section{INTRODUCCIÓN}

\section{Arteria temporal superficial}

Anatómicamente la arteria temporal superficial (ATS) es una arteria ampliamente descrita, ${ }^{1}$ proveniente de la arteria carótida externa, la ATS se origina en el espesor de la glándula parótida y se dirige hacia arriba y un poco hacia afuera, posteriormente se desprende de la glándula, se hace superficial a la altura de una línea trazada desde el borde superior del conducto auditivo externo a la parte media del borde superior de la órbita y continúa su ascenso pasando por delante del trago y por detrás de la articulación temporomandibular, emitiendo cinco ramas colaterales en su trayecto: la arteria facial transversa, la arteria auricular, la arteria temporal profunda posterior, la rama frontal o anterior y la rama parietal o posterior.

\section{Pseudoaneurismas de la arteria temporal superficial}

Desde que fue descrito en 1740 por Thomas Bartholin, ${ }^{1}$ menos de 400 casos de pseudoaneurismas de la arteria temporal superficial (PATS) han sido reportados en la literatura.

Se han descrito pseudoaneurismas en la arteria facial, maxilar interna, supraorbitaria y ATS. El factor común entre estos vasos que los predispone a la formación de pseudoaneurismas es que en algún punto de su trayecto se acercan a la superficie cruzando sobre prominencias óseas. La ATS es más vulnerable al trauma en su rama anterior, específicamente en el segmento que cruza sobre la zona de unión de la fascia temporal con la línea temporal superior. ${ }^{2}$ En general, alrededor de $80 \%$ del total de aneurismas de la ATS ocurren en hombres de entre 20 y 40 años.

Los pseudoaneurismas son anormalidades vasculares originadas cuando se da una disrupción en la continuidad de la pared arterial, causadas por inflamación, trauma, iatrogenia o por procedimientos quirúrgicos; cabe señalar que la etiología traumática es la más frecuente. La mayoría de los pacientes presentan el pseudoaneurisma dentro del rango de dos a seis semanas posteriores al trauma. Una fístula arteriovenosa puede también desarrollarse debido al involucramiento de la vena adyacente. ${ }^{3}$

La disrupción de la pared permite la extravasación y la formación de un "saco" lleno de sangre, que se encapsula por la adventicia arterial o por tejido subcutáneo y forma una pseudocápsula que reemplaza la pared arterial. La reabsorción del coágulo y el crecimiento a expensas de la fuerza pulsátil de la sangre se considera como el mecanismo fisiopatológico de su desarrollo. En las raras ocasiones en las que se han descrito aneurismas verdaderos con conservación de las capas arteriales son de origen ateroesclerótico por vasculitis e incluso postraumáticos. ${ }^{3}$
El cuadro clínico de esta patología se caracteriza por la aparición progresiva de un aumento de volumen en la región temporal, precedida usualmente por un traumatismo en dicha región de dos a seis semanas previas; sin embargo, existen reportes de aneurismas tardíos con presentación hasta de ocho años después del traumatismo. ${ }^{4}$ Clínicamente aparece como una masa quística pulsátil en la región de la frente y debe sospecharse si aparece poco después de un trauma contuso. Dicha tumoración tiende a ser pulsátil, comprimible, no dolorosa, de bordes regulares, que tiende a disminuir de tamaño al oprimir la ATS proximal. La pulsatilidad puede estar ausente en aquellos casos en los que el saco aneurismático ha sido completamente trombosado. Frémito o murmullo sugiere una fístula arteriovenosa. La compresión de la región proximal de la arteria temporal superficial debería resultar en una disminución o ausencia de pulsación en el aneurisma distal. La expansión gradual de la lesión aumenta el índice de sospecha. ${ }^{5}$ El síntoma acompañante más frecuente es una cefalea pulsátil hemicránea ipsilateral, pero puede ocasionar también alteraciones visuales, mareo o dolor en región auricular. En caso de ruptura puede ocasionar hemorragia masiva o edema hemifacial severo. ${ }^{6}$

Los pacientes posiblemente consulten a un dermatólogo, cirujano plástico o neurocirujano. Esta entidad rara puede ser confundida con un quiste de inclusión o inclusive lipoma, así que los diagnósticos diferenciales y las investigaciones especiales son imprescindibles si se intenta cualquier tratamiento curativo. ${ }^{7}$

Independiente del cuadro de presentación, el diagnóstico debe establecerse con base en la historia y examen físico apoyado por una evaluación diagnóstica. El ultrasonido Doppler es la primera línea de abordaje con un alto grado de sensibilidad. Herramientas como la tomografía (TC) y la resonancia magnética (RM) pueden ayudar a establecer el diagnóstico diferencial. La angiografía está reservada en casos especiales o cuando se desea el tratamiento endovascular. Es de utilidad llevar a cabo un estudio confirmatorio de imagen como pueden ser un ultrasonido Doppler, en el que se observa el signo de Yin-Yang, una angio-TC o angio$\mathrm{RM}$, en las que se puede observar el trayecto vascular que nos permite excluir los posibles diagnósticos diferenciales como aneurismas, tumores, hematoma subcutáneo, fístula arteriovenosa, lipoma, meningocele o un neuroma del nervio facial, entre otros. ${ }^{8}$

\section{CASO CLÍNICO}

Se trata de paciente masculino de 37 años, quien ingresa a la consulta de angiología y cirugía vascular el día 22 de octubre de 2019, originario de Cuauhtémoc, Chihuahua; 


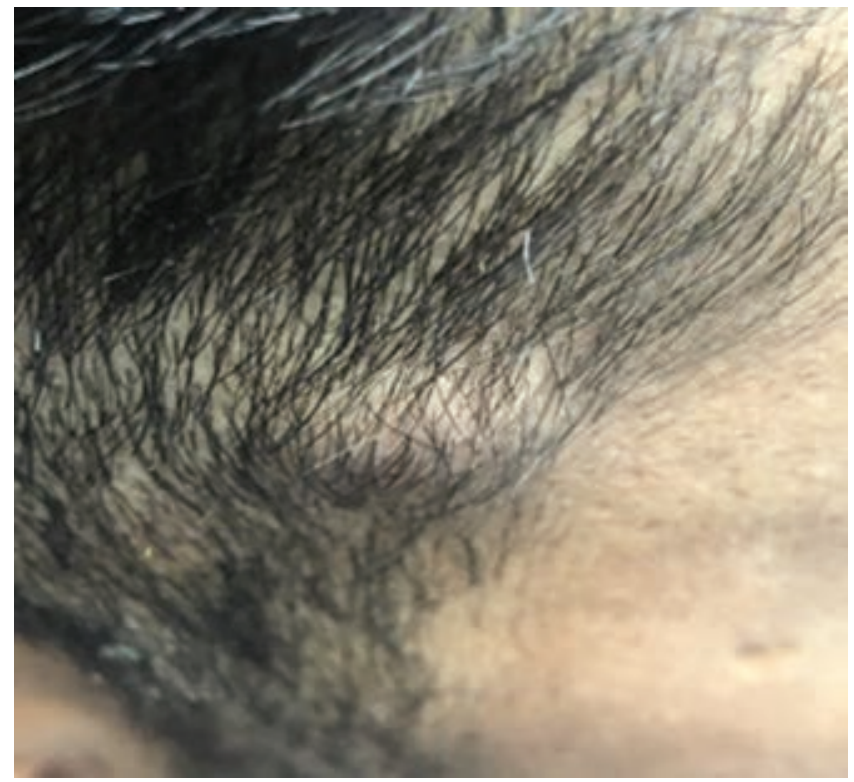

Figura 1: Masa pulsátil a nivel de trayecto ATS derecha, la cual en una exploración vascular no muestra presencia de frémito o soplos en dicho trayecto, con diámetro aproximado de 1-2 cm, no dolorosa y desplazable.

ocupación: constructor, sin antecedentes de importancia. Inicia su padecimiento tres meses antes de su interrogatorio, en el mes de julio de 2019, posterior a una agresión por terceras personas sufre golpe a nivel de región frontoparietal derecha con posterior aparición de masa pulsátil indolora, la cual fue progresando de manera paulatina. Dicha masa se encuentra en el trayecto de la ATS, la cual como sintomatología específica únicamente presenta aumento de volumen y dolor ocasional 3/10 en la escala visual análoga
(EVA) del dolor, motivo por el que acude con médico general, quien refiere al paciente al servicio de angiología para su valoración y tratamiento.

A la exploración física el paciente se encontraba alerta y orientado, con regular estado de hidratación de mucosas y tegumentos. Presencia de masa pulsátil a nivel de trayecto ATS derecha, la cual en una exploración vascular no muestra presencia de frémito o soplos en el trayecto, con diámetro aproximado de 1-2 cm (Figura 1), no dolorosa, desplazable. Se realiza rastreo con Doppler lineal y se encuentra flujo bifásico a nivel de dicha masa. Cuello cilíndrico sin megalias, tráquea central, pulsos carotídeos presentes de buena intensidad sin soplos, sincrónicos con latido cardiaco, exploración de tórax y abdomen sin alteraciones, extremidades torácicas con pulso axilar, braquial, radial y cubital de buena intensidad, presentes, normales, sincrónicos con latido cardiaco, extremidades pélvicas con pulso femoral, poplíteo, pedio y tibial posterior presentes, normales y sincrónicos con latido cardiaco. Llenado capilar conservado, normotermia, coloración normal y arcos de movilidad conservados en las cuatro extremidades. Citometría hemática, química sanguínea y electrolitos séricos dentro de los parámetros normales, y perfil viral negativo.

Posterior a ello se inicia protocolo diagnóstico, por lo que se solicita angiotomografía de cabeza y cuello con contraste intravenoso (Figura 2 A y B) y reconstrucción 3D (Figura 2C), en el que se visualiza disrupción de ATS derecha con paso de contraste a dicha disrupción, sin datos de fuga, por lo que se confirma como diagnóstico de PATS no roto.

\section{Procedimiento quirúrgico}

Bajo protocolo de sala ingresa paciente a sala de operaciones, previa intubación orotraqueal y con anestesia general
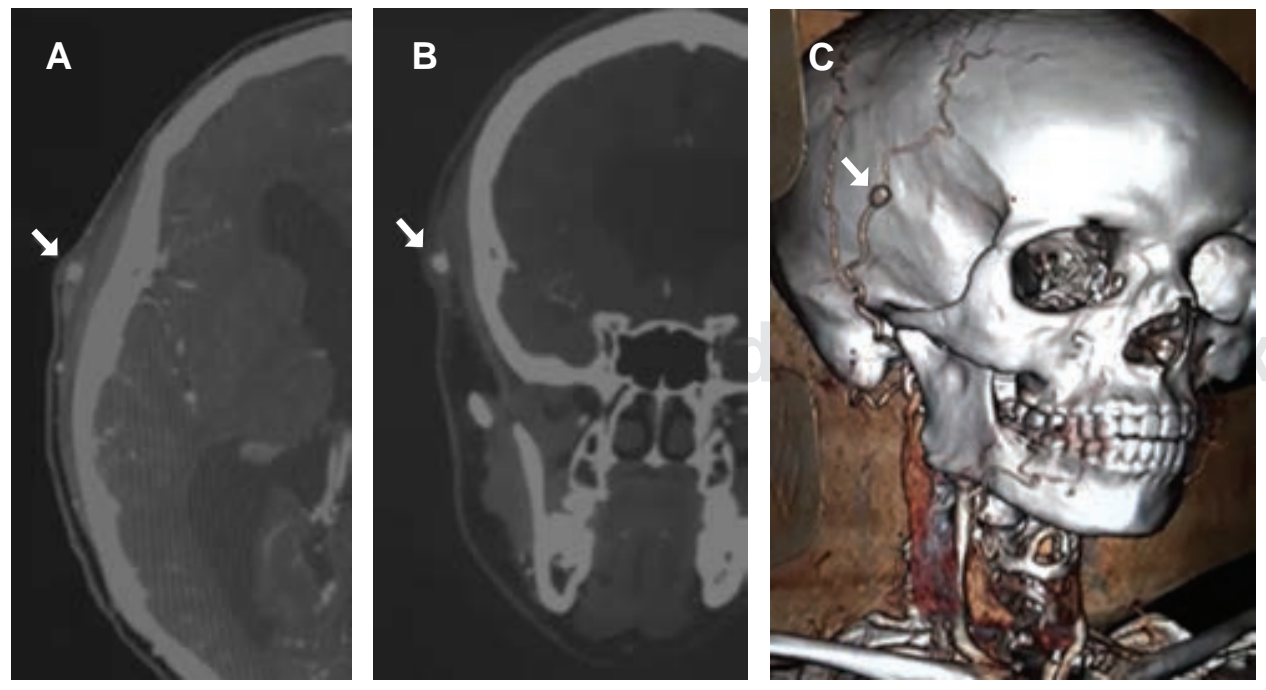

Figura 2:

Imagen de angiotomografía de cabeza y cuello con contraste intravenoso.

A) Corte transversal. B) Corte coronal. C) Reconstrucción 3D, se visualiza disrupción de ATS derecha (flecha roja), con paso de contraste a dicha disrupción sin datos de fuga. 

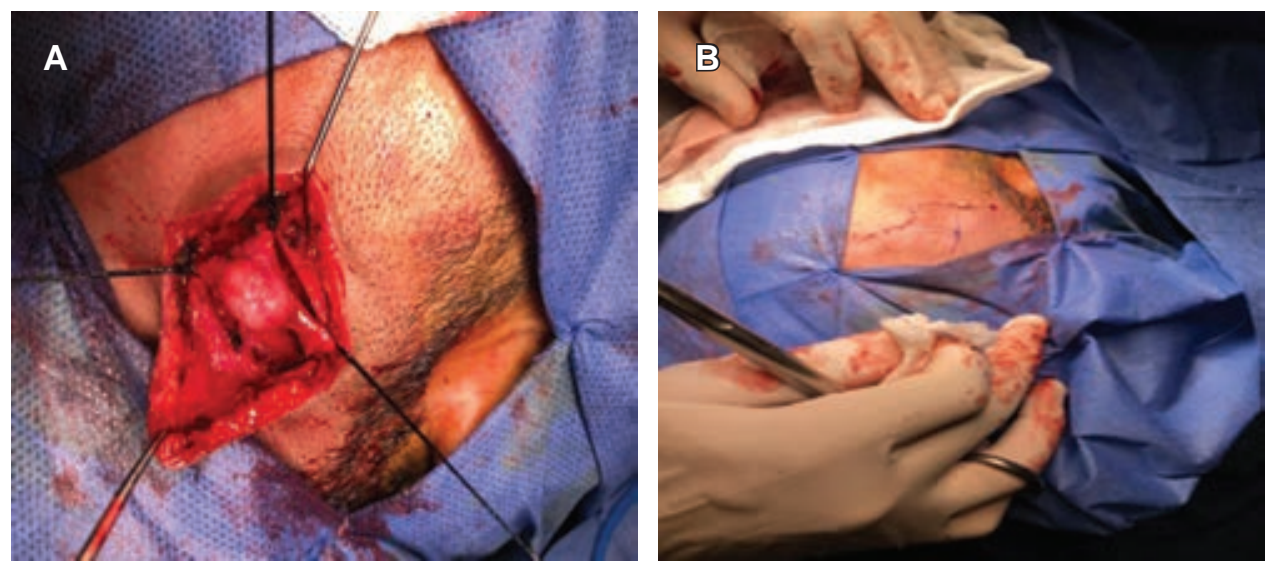

Figura 3:

Imágenes transoperatorias. A) Se visualiza PATS, disecado y con exclusión del mismo. B) Se visualiza cierre de piel con punto subdérmico.

balanceada se colocan campos estériles, posteriormente se localiza masa pulsátil en región temporal derecha, se realiza incisión en forma de $\mathrm{S}$-itálica de $7 \mathrm{~cm}$, se diseca por planos, piel, tejido celular subcutáneo, hasta localizar masa pulsátil dependiente de la arteria temporal superficial, se realiza disección proximal y distal a la masa, se identifican ATS proximal y distal a la masa de la rama frontal, la cual se bifurca en una rama anterior y otra posterior, se realiza disección de los ramos identificados, se refieren y posteriormente se ligan con seda 2-0 (Figura 3A), se realiza exclusión de pseudoaneurisma de arteria temporal superficial, el cual se reseca y se envía a histopatología, después se realiza hemostasia con electrocauterio, se afronta tejido celular subcutáneo con Vicryl 3-0, piel con punto subdérmico con Prolene 3-0 (Figura 3B).

\section{DISCUSIÓN}

Los PATS son una entidad poco frecuente y hasta cierto punto subdiagnosticada, ya que dentro de sus diagnósticos diferenciales existen una gran cantidad de enfermedades relacionadas en esta localización, además de ello es importante tener en cuenta el antecedente relacionado a traumatismo descrito por Thomas Bartholin desde el siglo XVIII.

En el caso se expone a un paciente masculino de 37 años de edad con antecedente de agresión por terceras personas, dicha agresión se localiza a nivel de hueso temporal que, como lo describe la literatura, aumenta el riesgo de sufrir algún tipo de disrupción de la arteria y por consiguiente aumenta el riesgo de la formación de una cápsula por un hematoma, el cual en el transcurso de dos a seis semanas (justo como ocurrió con el paciente a las tres semanas) el hematoma se vuelve pulsátil y de esta manera pasa a ser una saco, el cual contiene la sangre, encapsulando la adventicia de la arteria y a la vez se forma una pseudocápsula, y esto es a lo que refiere un PATS.
Hoy en día, su incidencia es relativamente baja, hay que tener en cuenta varios factores al momento de evaluar a un paciente con un tumor a nivel del temporal, regularmente son pacientes entre la segunda y la cuarta década de la vida, varones, que tienen como antecedente hasta en $80 \%$ de los casos un traumatismo en la región. Al explorar a un paciente y clínicamente observar un hematoma pulsátil a nivel del temporal, se debe sospechar de un pseudoaneurisma y de esta manera protocolizarlo a pesar de no ser una entidad frecuente para su respectivo diagnóstico, que de manera estandarizada se puede iniciar con estudios poco invasivos como el ultrasonido Doppler, y una vez que la sospecha clínica y por imagen sea mayor, la angiografía es considerada el "estándar de oro" o la angiotomografía, tal como se realizó con el paciente en cuestión, ya que ésta cuenta con sensibilidad y especificidad alta debido a su utilidad para excluir los diversos diagnósticos diferenciales. El paciente una vez diagnosticado fue sometido a exploración vascular de la región temporal y se realizó ligadura de cabos proximales y distales. En la actualidad, es uno de los tratamientos más empleados para dicha patología; sin embargo, la elección del tratamiento de primera línea debe ser siempre individualizado.

Las opciones de tratamiento en los pseudoaneurismas y aneurismas verdaderos varían desde el tratamiento expectante, compresión con ultrasonido hasta escisión quirúrgica con ligadura. Otras opciones terapéuticas incluyen la obliteración endovascular y la inyección percutánea de trombina. Cada opción de tratamiento está asociada con riesgos y requieren consideraciones cuidadosas. El propósito principal es reducir el riesgo de hemorragia y también mejorar los aspectos estéticos. Sólo hasta hace poco las alternativas endovasculares han sido consideradas como una modalidad terapéutica atractiva. ${ }^{9}$

A pesar de las múltiples opciones terapéuticas disponibles, el uso de la embolización endovascular está ganando popularidad para lesiones de la arteria temporal superficial, 
principalmente en la porción proximal, debido a que, por lo general, la resección quirúrgica necesita disección de la glándula parótida y con ello una lesión potencial al nervio. Abordajes endovasculares han sido usados en 13\% de los pseudoaneurismas diagnosticados, con una tasa de éxito significativa. La opción de resortes metálicos en el tratamiento endovascular ha sido reportada como una opción ideal para la embolización de pseudoaneurismas de la ATS. ${ }^{10}$

Invariablemente el PATS no es una entidad común; sin embargo, el médico general debe conocerla y tenerla en su repertorio de diagnósticos diferenciales para poder ofrecer de manera oportuna el tratamiento y evitar complicaciones como hemorragia, fístula arteriovenosa y problemas estéticos relacionados.

\section{REFERENCIAS}

1. Rouvière H, Delmas A. Anatomía humana. Descriptiva, topográfica y funcional. Tomo 1. 9a. ed. Ed. Masson S.A. USA; 1994, 214-215.

2. Conner W, Rohrich R, Pollock R. Traumatic aneurysms of the face and temple: a case report and literature review. Ann Plast Surg. 1998; 41 (3): $321-326$
3. Tarud R, Fourzali R, Aycardi R, Mercado G, Sabbag J. Pseudoaneurisma de la arteria temporal superficial diagnosticado por ultrasonido Doppler: reporte de caso. Rev Colomb Radiol. 2011; 22(2): 32063208.

4. Khandelwal P, Akkara F, Dhupar V, Louis A. Traumatic pseudoaneurysm of the superficial temporal artery. Natl J Maxillofac Surg. 2018; 9 (1): 74-77.

5. Gull S, Badawy A, Chaudhuri A. The pulsatile sebaceous cyst: beware of a superficial temporal artery aneurysm. BMJ Case Rep. 2009; 2009: bcr03.2009.1698.

6. Khandelwal P, Akkara F, Dhupar V, Louis A. Traumatic pseudoaneurysm of the superficial temporal artery. Natl J Maxillofac Surg. 2018; 9 (1): 74-77.

7. Cohen JE, Itshayek E. Traumatic pseudoaneurysm of the superficial temporal artery after paintball injury. Isr Med Assoc J. 2010; 12 (2): 123-124.

8. Burleson SL, Cirillo FN, Gibson CB, Gullett JP, Pigott DC. Superficial temporal artery pseudoaneurysm diagnosed by point-of-care ultrasound. Clin Pract Cases Emerg Med. 2019; 3 (1): 77-78.

9. Shenoy SN, Raja A. Traumatic superficial temporal artery aneurysm: case report. Neurol India. 2003; 51 (4): 537-538.

10. Park SK, Bong HJ, Jang DK, Yoon WS, Han YM, Jang KS. Traumatic true aneurysm of the superficial temporal artery. Aesthetic Plast Surg. 2012; 36 (4): 934-937.

Conflicto de intereses: Los autores declaran que no existe conflicto de intereses. 